\title{
Effects of cycle patterns of accelerated crucible rotation technique (ACRT) on the flows, interface, and segregation in vertical Bridgman crystal growth
}

\author{
Y.C. Liu ${ }^{a}$, B. Roux ${ }^{b}$, C.W. Lan ${ }^{a} *$ \\ ${ }^{a}$ Department of Chemical Engineering, National Taiwan University, Taipei 10617, Taiwan, ROC \\ ${ }^{\mathrm{b}}$ Laboratoire Modélisation et simulation numérique en mécanique, L3M: CNRS-Universités d'Aix-Marseille, France
}

Received 17 January 2007; received in revised form 17 July 2007

Available online 22 October 2007

\begin{abstract}
The accelerated crucible rotation technique (ACRT) has been used extensively in crystal growth for the control of flow and segregation. However, the amelioration of the spin-up and spin-down flows for a favorable growth has not yet been fully understood. In this study, we investigate several ACRT schemes and discuss their effects on the flow, interface morphology, and solute segregation during the vertical Bridgman growth of succinonitrile (SCN) containing acetone. Both numerical simulation and visualization experiments are conducted. In addition to the triangular and trapezoidal patterns, stair-up, stair-down, and pulse schemes are proposed for a better flow and mixing control without much interface deformation. For all the ACRT schemes investigated here, the simulated results are in good agreement with the observed ones.
\end{abstract}

(C) 2007 Elsevier Ltd. All rights reserved.

PACS: 44.25.+f; 47.27.Te; 81.10.Fq; 02.60.c6; 02.70.Fj

Keywords: ACRT; Segregation; Cycle pattern; Convection; Bridgman crystal growth

\section{Introduction}

The control of interface morphology and segregation is important in the crystal growth. Especially for the vertical Bridgman (VB) process, due to a lack of control over the stirring condition, the introduction of forced convection to control the interface morphology and solute mixing is necessary. Scheel and Schulz-Dubois [1] first introduced the acceleration crucible rotation technique (ACRT) to enhance the melt flow during VB crystal growth. Over the years, ACRT has been widely used, and the improvement of crystal quality has been reported [2-8]. To reduce both radial and axial segregations, a careful flow control

\footnotetext{
${ }^{*}$ Corresponding author. Tel./fax: +886 23633917.

E-mail address: cwlan@ntu.edu.tw (C.W. Lan).
}

near the interface is necessary, while the bulk mixing needs to be minimized for the reduction of axial segregation. However, the amelioration of the Ekman flow is not trivial, and its interactions with buoyancy convection and TaylorGörtler instability $[10,11]$ often makes the flow control complicated. Indeed, there are a number of parameters in using ACRT, but the most important parameter is the cycle period and patterns. As a general rule, in order to develop the Ekman boundary layer, the time for a spin-up or spindown cycle is usually in the order of $2 R_{\mathrm{C}} / \sqrt{\Omega_{\mathrm{R}} v_{\mathrm{m}}}[10,11]$, where $R_{\mathrm{C}}$ is the crystal radius, $\Omega_{\mathrm{R}}$ the rotation amplitude, and $v$ the kinematic viscosity of the melt. This time constant, as a quarter of the cycle period, is usually several seconds for most of the materials having a diameter of several centimeters. In practice, the triangular and trapezoidal cycle patterns are often adopted $[3,9,12]$. Beside the simplicity, using these ACRT schemes are based on empirical 


\begin{tabular}{|c|c|c|c|}
\hline \multicolumn{4}{|c|}{ Nomenclature } \\
\hline$C$ & solute concentration & $u_{\mathrm{t}}$ & tangential velocity at the Schlichting boundary \\
\hline$C_{p}$ & specific heat & & layer \\
\hline$D$ & solute diffusivity in the melt & $v$ & $z$-component of velocity \\
\hline $\boldsymbol{e}_{z}$ & unit vector in $z$-direction & $v_{\theta}$ & azimuthal velocity \\
\hline$G$ & thermal gradient & $z$ & cylindrical coordinate \\
\hline$g_{0}$ & gravitational acceleration & & \\
\hline$\Delta H$ & heat of fusion & \multicolumn{2}{|c|}{ Greek symbols } \\
\hline$h$ & heat transfer coefficient & $\alpha$ & thermal diffusivity \\
\hline$h_{\mathrm{c}}$ & height of growth front & $\beta_{\mathrm{T}}$ & thermal expansion coefficient \\
\hline$k$ & thermal conductivity & $\beta_{\mathrm{S}}$ & solutal expansion coefficient \\
\hline$K$ & equilibrium segregation coefficient & $\kappa v$ & kinematic viscosity, $\mu_{\mathrm{m}} / \rho_{\mathrm{m}}$ \\
\hline$L$ & length of melt & $\mu$ & viscosity \\
\hline$m$ & slope of the liquidus line in the phase diagram & $\rho$ & density \\
\hline$n$ & unit normal vector & $\gamma$ & ratio of crystal and melt density, $\rho_{\mathrm{c}} / \rho_{\mathrm{m}}$ \\
\hline $\operatorname{Pr}$ & Prandtl number, $v_{\mathrm{m}} / \alpha_{\mathrm{m}}$ & $\Psi$ & stream function \\
\hline$r$ & cylindrical coordinate & $\omega$ & stream function \\
\hline$R a_{\mathrm{T}}$ & thermal Rayleigh number, $\beta_{\mathrm{T}} R_{\mathrm{c}}^{3} g_{0} T_{\mathrm{m}} / v_{\mathrm{m}} \alpha_{\mathrm{m}}$ & $\Omega$ & rotation speed \\
\hline$R a_{\mathrm{S}}$ & solutal Rayleigh number, $\beta_{\mathrm{s}} R_{\mathrm{c}}^{3} g_{0} C_{0} / v_{\mathrm{m}} \alpha_{\mathrm{m}}$ & & \\
\hline$R_{\mathrm{c}}$ & radius of crystal & \multicolumn{2}{|c|}{ Superscript } \\
\hline$t$ & time & $*$ & dimensionless variables \\
\hline$S c$ & Schmidt number, $v_{\mathrm{m}} / D$ & & \\
\hline St & Stefan number, $\Delta H /\left(C_{p} \mathrm{~m} T_{\mathrm{m}}\right)$ & \multicolumn{2}{|c|}{ Subscripts } \\
\hline$T$ & temperature & 0 & initial value \\
\hline$T a$ & Taylor number, $4 \Omega^{2} R_{\mathrm{c}}^{4} /\left(\mu_{\mathrm{m}} / \rho_{\mathrm{m}}\right)^{2}$ & $\mathrm{a}$ & ampoule \\
\hline$T_{\mathrm{C}}$ & temperature of cold-zone & $\mathrm{c}$ & crystal \\
\hline$T_{\text {eff }}$ & effective heater temperature & $\mathrm{h}$ & heater \\
\hline$T_{\mathrm{H}}$ & temperature of hot-zone & $\mathrm{m}$ & melt \\
\hline$T_{\mathrm{m}}$ & melting point & $\max$ & maximum \\
\hline$U$ & translation speed & $\min$ & minimum \\
\hline$u$ & $r$-component of velocity & $\mathrm{R}$ & radial direction \\
\hline
\end{tabular}

results and hydrodynamic considerations. However, the enhancement of melt mixing near the interface is not adequate for a good growth control. In addition to the control of local flow favoring the uniform solute composition, the reduction of interface concavity and growth striations is also important. However, the optimization of the cycle patterns to meet these requirements has not yet been reported.

In our previous work [13], a triangular ACRT scheme was considered for the VB growth of SCN containing $0.064 \mathrm{wt} \%$ acetone. Through the observation of the interface shape and the morphological breakdown pattern, the effect of rotation speed was investigated, where the ACRT was implemented on a rotating table. Indeed, with the amplitude of 60 RPM, the morphological breakdown was suppressed [13]. Based on the numerical simulation, the radial segregation was found significantly reduced. However, the growth interface was very concave as a result of the averaged Ekman flow that enhances the heat transfer toward the interface. In this study, we attempt to modify the cycle patterns that can reach the same composition uniformity and morphology stability, but without too much interface deformation. Also, to precisely implement the
ACRT schemes, a micro-stepping motor is used to rotate the ampoule directly. Computer simulation is also carried out and the simulated results are compared with the experimental observations.

In the next section, experimental setup and procedure are described briefly. The numerical simulation is discussed in Section 3. Section 4 is devoted to results and discussed, followed by conclusions in Section 5 .

\section{Crystal growth and interface visualization}

Succinonitrile (SCN) containing $0.064 \mathrm{wt} \%$ acetone was directionally solidified in a transparent vertical Bridgman system as shown in Fig. 1. Before experiments, high-purity SCN was required. SCN (ACROS, about $99 \%$ purity) was purified first by vacuum distillation at $50 \mathrm{mtorr}$ for five times. The distilled sample, collected in a $17-\mathrm{mm}$ diameter Pyrex ampoule ( $2.5 \mathrm{~mm}$ in thickness), was further purified by three-zone refining for more than 60 passes. The purified sample was then examined by directional solidification. No morphological breakdown was observed up to a solidification speed of $7 \mu \mathrm{m} / \mathrm{s}$ at a thermal gradient of $8-10 \mathrm{~K} / \mathrm{cm}$. 


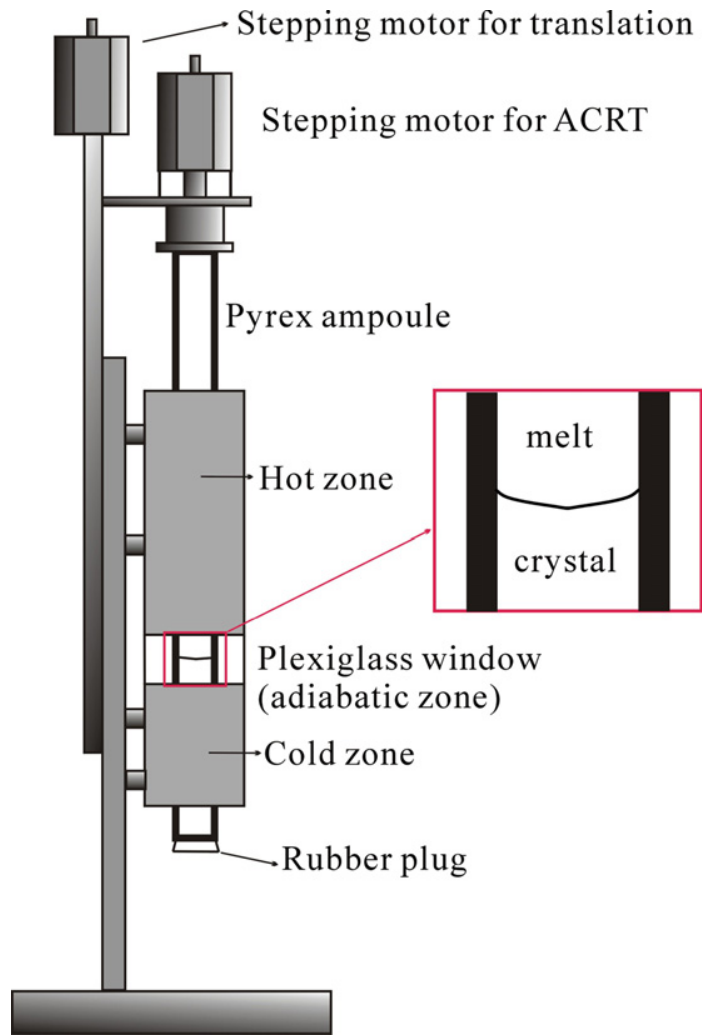

Fig. 1. Experimental setup for vertical Bridgman crystal growth with ACRT steeping motor driving upon the crucible.

To perform crystal growth experiments, about $0.064 \mathrm{wt} \%$ of acetone was injected into the sample through a $5 \mu 1$ micro-syringe inserted into the bottom of the sample. The total sample length was about $20 \mathrm{~cm}$. A stationary state was achieved by keeping the hot-zone at $80^{\circ} \mathrm{C}$ and coldzone $40^{\circ} \mathrm{C}$, respectively, for $24 \mathrm{~h}$ to make sure that the acetone was well mixed.

A stepping motor was used to rotate the crucible directly. Through the computer-controlled interface, an arbitrary ACRT cycle pattern can be easily generated. Five cycle patterns, as shown in Fig. 2, were considered in this study. The triangular and trapezoidal patterns shown in Fig. $2 \mathrm{a}$ and $\mathrm{b}$ have been widely adopted. The new patterns,

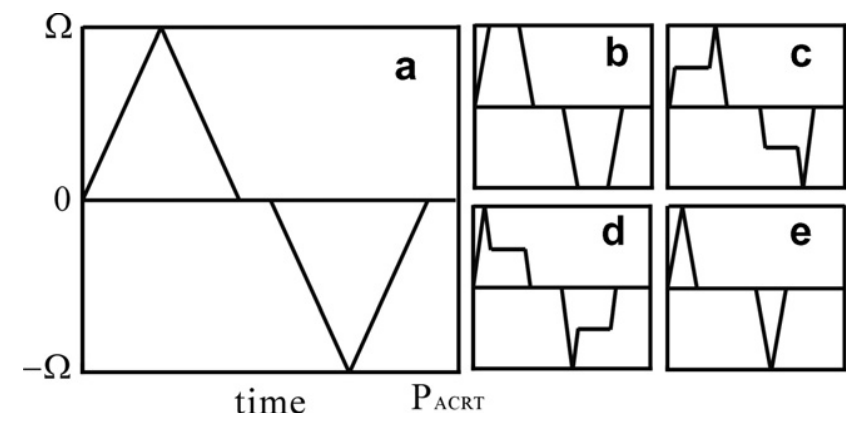

Fig. 2. Illustrations for conventional and new wave types of ACRT in one period. (a) triangular wave, (b) square wave, (c) stair-up wave, (d) stairdown wave, and (e) pulse wave. the stair-up (Fig. 2c), stair-down (Fig. 2d), and pulse (Fig. 2e), are the newly proposed ones here. The crucible was rotated based on the given pattern right after the ampoule was translated. A digital camera (Nikon D-50) was used to capture the interface shape during crystal growth.

\section{Computer simulations}

Computer simulation is further used to study the convection, heat and mass transfer, and the interface shape in the VB growth of SCN containing acetone. The furnace environment used for simulation is described by an effective heating profile $T_{\text {eff }}(z, t)$, which is specified in modeling. To start crystal growth from a stationary state (without ampoule movement), this profile is moved upward at speed $U_{\mathrm{h}}$. In the experiments, the furnace was kept stationary, while the ampoule was moving downward at a given speed after the growth started. The system is assumed axisymmetric, so that the flow and temperature fields, as well as the growth front (the melt/crystal interface, $h_{\mathrm{c}}(r, t)$ ). The melt is further assumed incompressible and Newtonian, while the flow is laminar. The Boussinesq approximation is also adopted. Dimensionless variables are defined by scaling length with the crystal radius $R_{\mathrm{c}}$, time $t$ with $R_{\mathrm{c}}^{2} / \alpha_{\mathrm{m}}$, velocity with $\alpha_{\mathrm{m}} / R_{\mathrm{c}}$, temperature with the melting point $T_{\mathrm{m}}$, and dopant or solute concentration by the initial concentration $C_{0}$, where $\alpha_{\mathrm{m}}$ is the thermal diffusivity of the melt. For the convenience of representation, all the variables defined afterwards with a superscript $*$ are dimensionless unless otherwise stated. The governing equations for the timedependent fluid flow and heat and mass transfer in terms of dimensionless stream function $\Psi^{*}$, vorticity $\omega^{*}$, azimuthal velocity $v_{\theta}^{*}$, temperature $T^{*}$, and dopant concentration $C^{*}$ can be written as the following:

\section{Equation of motion}

$$
\begin{aligned}
& \frac{\partial \omega^{*}}{\partial t^{*}}+\frac{\partial}{\partial r^{*}}\left(\frac{\omega^{*}}{r^{*}} \frac{\partial \psi^{*}}{\partial z^{*}}\right)-\frac{\partial}{\partial z^{*}}\left(\frac{\omega^{*}}{r^{*}} \frac{\partial \psi^{*}}{\partial r^{*}}\right) \\
& +\operatorname{Pr}\left[\frac{\partial}{\partial r^{*}}\left(\frac{1}{r^{*}} \frac{\partial}{\partial r^{*}}\left(r^{*} \omega^{*}\right)\right)+\frac{\partial}{\partial z^{*}}\left(\frac{1}{r^{*}} \frac{\partial}{\partial z^{*}}\left(r^{*} \omega^{*}\right)\right)\right] \\
& +\frac{\partial}{\partial z}\left(\frac{v_{\theta}^{2}}{r}\right)-\operatorname{Pr} R a_{\mathrm{T}} \frac{\partial T^{*}}{\partial r^{*}}+\operatorname{Pr} R a_{S} \frac{\partial C^{*}}{\partial r^{*}}=0 .
\end{aligned}
$$

\section{Stream equation}

$$
\frac{\partial}{\partial z^{*}}\left(\frac{1}{r^{*}} \frac{\partial \psi^{*}}{\partial z^{*}}\right)+\frac{\partial}{\partial r^{*}}\left(\frac{1}{r^{*}} \frac{\partial \psi^{*}}{\partial r^{*}}\right)+\omega^{*}=0 .
$$

\section{Circulation equation}

$$
\begin{aligned}
& -\frac{\partial v_{\theta^{*}}}{\partial t^{*}}+\frac{1}{r^{*^{2}}}\left[\frac{\partial}{\partial r^{*}}\left(r^{*} v_{\theta}^{*} \frac{\partial \psi^{*}}{\partial z^{*}}\right)-\frac{\partial}{\partial z^{*}}\left(r^{*} v_{\theta}^{*} \frac{\partial \psi^{*}}{\partial r^{*}}\right)\right] \\
& +\operatorname{Pr}\left[\frac{\partial}{\partial r^{*}}\left(\frac{1}{r^{*}} \frac{\partial\left(r^{*} v_{\theta}^{*}\right)}{\partial r^{*}}\right)+\frac{\partial}{\partial z^{*}}\left(\frac{1}{r^{*}} \frac{\partial\left(r^{*} v_{\theta}^{*}\right)}{\partial z^{*}}\right)\right]=0 .
\end{aligned}
$$




\section{Energy equation}

$$
\begin{aligned}
& -r^{*} \frac{\partial T^{*}}{\partial t^{*}}-\frac{\partial}{\partial r^{*}}\left(r^{*} u^{*} T^{*}\right)-\frac{\partial}{\partial z^{*}}\left(r^{*} v^{*} T^{*}\right) \\
& +\frac{\partial}{\partial z^{*}}\left(r^{*} \alpha_{i}\left(T^{*}\right) \frac{\partial T^{*}}{\partial z^{*}}\right)+\frac{\partial}{\partial r^{*}}\left(r^{*} \alpha_{i}\left(T^{*}\right) \frac{\partial T^{*}}{\partial r^{*}}\right)=0, \\
& i=(\mathrm{m}, \mathrm{c}, \mathrm{amp}) .
\end{aligned}
$$

Solute equation

$$
\begin{aligned}
- & r^{*} \frac{\partial C^{*}}{\partial t^{*}}-\frac{\partial}{\partial r^{*}}\left(r^{*} u^{*} C^{*}\right)-\frac{\partial}{\partial z^{*}}\left(r^{*} v^{*} C^{*}\right) \\
+ & \frac{P r}{S c}\left[\frac{\partial}{\partial z^{*}}\left(r^{*} \frac{\partial C^{*}}{\partial z^{*}}\right)+\frac{\partial}{\partial r^{*}}\left(r^{*} \frac{\partial C^{*}}{\partial r^{*}}\right)\right]=0 .
\end{aligned}
$$

The solute diffusion in the solid phase is neglected. In the above equations, $P r$ is the Prandtl number $\left(\operatorname{Pr} \equiv v_{\mathrm{m}} / \alpha_{\mathrm{m}}\right)$, where $v_{\mathrm{m}}$ is the kinematic melt viscosity), $S c$ the Schmidt number $\left(S c \equiv v_{\mathrm{m}} / D\right)$, and $D$ the solute diffusivity in the melt. Also, $\alpha_{i}$ is the thermal diffusivity of phase $i ; i=\mathrm{c}$ for the crystal, $m$ for the melt, and amp for the ampoule. Two important dimensionless variables, $R a_{\mathrm{T}}$ and $R a_{\mathrm{S}}$, in the source term of the equation of motion are defined as follows:

$R a_{\mathrm{T}} \equiv \frac{g_{0} \beta_{\mathrm{T}} T_{\mathrm{m}} R_{\mathrm{C}}^{3}}{\alpha_{\mathrm{m}} v_{\mathrm{m}}} ; \quad R a_{\mathrm{S}} \equiv \frac{g_{0} \beta_{\mathrm{S}} C_{0} R_{\mathrm{C}}^{3}}{\alpha_{\mathrm{m}} v_{\mathrm{m}}}$,

where $g_{0}$ is the gravitational acceleration and $\beta_{\mathrm{T}}$ and $\beta_{\mathrm{S}}$ are the thermal and solutal expansion coefficients, respectively. The stream function $\psi$ and vorticity $\omega$ in the above equations are defined in terms of the radial $(u)$ and axial $(v)$ velocities as

$u^{*}=-\frac{1}{r^{*}} \frac{\partial \psi^{*}}{\partial z^{*}}, \quad v^{*}=\frac{1}{r^{*}} \frac{\partial \psi^{*}}{\partial r^{*}}$,

$\omega^{*}=\frac{\partial u^{*}}{\partial z^{*}}-\frac{\partial v^{*}}{\partial r^{*}}$.

To solve the above governing equations, boundary conditions are also required. Most of the boundary conditions for melt flow and heat and mass transfer can be found elsewhere [14]. In short, the energy and solute conservation are applied to the growth interface. For example, at the melt/ crystal interface, the interface energy and solute balances can be written as the following, respectively,

$$
\begin{gathered}
\left.\boldsymbol{n} \cdot \nabla T^{*}\right|_{\mathrm{m}}-\left.\boldsymbol{n} \cdot \kappa_{\mathrm{c}} \nabla T^{*}\right|_{\mathrm{c}}+\left.\gamma S t \frac{\partial h_{\mathrm{c}}^{*}}{\partial t^{*}}\left(\boldsymbol{n} \cdot \boldsymbol{e}_{z}\right)\right|_{\mathrm{c}}=0, \\
-\left.\boldsymbol{n} \cdot \nabla C^{*}\right|_{\mathrm{c}}-\left.(1-K) \frac{S c}{P r} C^{*} \frac{\partial h_{\mathrm{c}}^{*}}{\partial t^{*}}\left(\boldsymbol{n} \cdot \boldsymbol{e}_{z}\right)\right|_{\mathrm{c}}=0,
\end{gathered}
$$

where $\boldsymbol{n}$ is the surface normal at the growth front pointing to the melt, $\kappa_{\mathrm{c}} \equiv k_{\mathrm{c}} / k_{\mathrm{m}}$ the normalized crystal thermal conductivity, $\gamma \equiv \rho_{\mathrm{c}} / \rho_{\mathrm{m}}$ the density ratio, and $h_{\mathrm{c}}^{*}$ the dimensionless interface height. The Stefan number $S t \equiv \Delta H /\left(C_{p_{\mathrm{m}}} T_{\mathrm{m}}\right)$ scales the heat of fusion $(\Delta H)$ released during solidification to the sensible heat in the melt. The equilibrium segregation coefficient $K \equiv C_{\mathrm{c}} / C$ is obtained according to the phase diagram; $K=0.1$ for acetone in $\mathrm{SCN}$. At the ampoule/material interfaces, heat flux conti-
Table 1

Physical properties and some input parameters for numerical simulations

SCN/Acetone

$\rho_{\mathrm{c}}=1.016 \mathrm{~g} \mathrm{~cm}^{-3}$

$\rho_{\mathrm{m}}=0.982 \mathrm{~g} \mathrm{~cm}^{-3}$

$T_{\mathrm{m}}=331.233 \mathrm{~K}$

$\Delta H=48.38 \mathrm{~J} \mathrm{~g}^{-1}$

$h=0.16 \mathrm{~W} \mathrm{~cm}^{-2} \mathrm{~K}^{-1}$

$k_{\mathrm{c}}=k_{\mathrm{m}}=2.24 \times 10^{-3} \mathrm{~W} \mathrm{~cm}^{-1} \mathrm{~K}^{-1}$

$C_{p_{\mathrm{c}}}=C_{p \mathrm{~m}}=2 \mathrm{~J} \mathrm{~g}^{-1} \mathrm{~K}^{-1}$

$\beta_{\mathrm{T}}=1.07 \times 10^{-3} \mathrm{~K}^{-1}$

$\beta_{\mathrm{S}}=1 \times 10^{-3}(\mathrm{wt} \% \text { acetone })^{-1}$

$D=1.3 \times 10^{-5} \mathrm{~cm}^{2} / \mathrm{s}$

$\mu_{\mathrm{m}}=2.6 \times 10^{-2} \mathrm{~g} \mathrm{~s}^{-1} \mathrm{~cm}^{-1}$

$K=0.1$

Ampoule

$\rho_{\mathrm{a}}=2.2 \mathrm{~g} \mathrm{~cm}^{-3}$

$k_{\mathrm{a}}=3.5 \times 10^{-2} \mathrm{~W} \mathrm{~cm}^{-1} \mathrm{~K}^{-1}$

$C_{p_{\mathrm{a}}}=0.188 \mathrm{~J} \mathrm{~g}^{-1} \mathrm{~K}^{-1}$

Other input parameters

$L=15 \mathrm{~cm}$

$R_{\mathrm{c}}=0.85 \mathrm{~cm}$

$R_{\mathrm{a}}=1.1 \mathrm{~cm}$

$T_{\mathrm{H}}=80^{\circ} \mathrm{C}$

$T_{\mathrm{C}}=40^{\circ} \mathrm{C}$

$G=10^{\circ} \mathrm{C} / \mathrm{cm}$

$U_{\mathrm{a}}=-1.6 \times 10^{-4} \mathrm{~cm} / \mathrm{s}$

$\Omega=60 \mathrm{RPM}$

Dimensionless groups

$P r=23.2142 ; S c=2.036 \times 10^{3} ; R a_{\mathrm{T}}=8.51 \times 10^{5} ; R a_{\mathrm{S}}=1.27 \times 10^{3} ;$

$T a=1.18 \times 10^{5}($ for $60 \mathrm{RPM})$

nuity is forced, while the solute flux is set to zero. The melt surface is set to stress free. The no-slip boundary condition is used for the solid boundaries of the interface. Therefore, the azimuthal velocity $v_{\theta}^{*}$, at the interface is related to the ACRT cycle pattern $\Omega^{*}\left(t^{*}\right)$ :

$v_{\theta}^{*}=\Omega^{*}\left(t^{*}\right) r^{*}$.

The above governing equations and boundary conditions are discretized by a finite volume method, and the resultant differential/algebraic equations are solved by DASPK solver with adaptive stepsize control [14]. The total number of unknowns after the finite volume approximation is 34,052 , and all the calculations are performed in a personal computer (P4-3 GHz CPU). The physical properties and corresponding dimensional groups used in the simulation are listed in Table 1.

In this study, the period of ACRT is fixed at $24 \mathrm{~s}$ in each cycle, which is based on the estimation of the Ekman time scale developing a spin-up flow (about $5 \mathrm{~s}$ at $60 \mathrm{RPM}$ ) [10].

\section{Results and discussion}

Before the results for the growth using ACRT are presented, we shall discuss the situation without rotation first. Fig. 3a shows the simulated flow (right) and acetone (left) fields, as well as the interface shape, after an hour of growth. As shown, two flow cells appear in the melt. The upper buoyancy cell is due to the side heating from the 


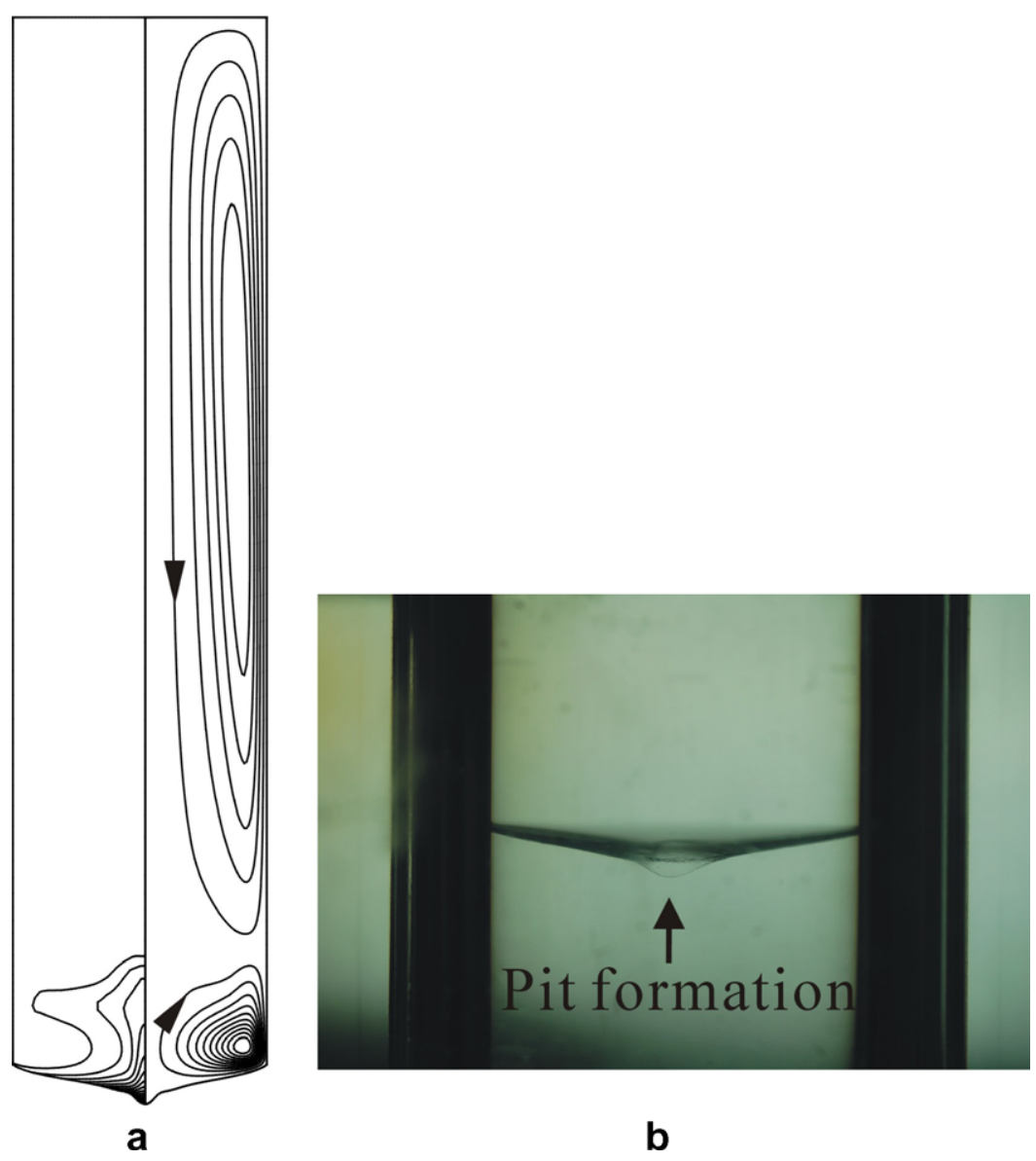

Fig. 3. Growth of SCN/acetone in the same conditions without ACRT for comparison: (a) simulated flow (right) and acetone (left) fields and (b) the observed interface shape after an hour of growth time; in the simulation, $\Psi_{\min }=-1.488 \times 10^{-4}, \Psi_{\max }=2.868 \times 10^{-4} \mathrm{~g} / \mathrm{s}$ and $C / C_{0}=0.993-8.474$.

furnace, while the lower cell is induced by the interface deformation as a result of the release of heat of fusion during crystal growth and the acetone segregation. As shown from the acetone field, the thermal convection delivers the rejected acetone from growth front to the center of the interface. This leads to a pit formation, again as a result of the acetone accumulation that lowers the solidification temperature. As discussed before [15-17], at stationary the interface was flat. As the growth started, the interface started to deform due to the latent heat. Meanwhile, the inward buoyancy flow was induced and the acetone was pushed toward the center of the interface. At about $30 \mathrm{~min}$, a depression at the interface center was observed. As the solidification continued, acetone accumulated further until the morphological breakdown, which was caused by constitutional supercooling, at about $40 \mathrm{~min}$. The cellular structures at the breakdown region trapped the acetone more and modified the segregation behavior. The observed interface corresponding to the results in Fig. 3a is shown in Fig. $3 \mathrm{~b}$ for comparison. As shown, they are in good agreement; a clear deep pit was formed and some morphological breakdown inside the pit was observed from the photograph.

As discussed in the previous report [13], the use of ACRT significantly reduced constitutional supercooling, so that the morphological breakdown could be avoided. This could be done by either reducing the radial acetone segregation or enhancing global acetone mixing. Although the later approach reduces the acetone accumulation at the growth front, the global mixing can lead to a larger axial segregation. The enhanced Ekman flow can also cause a large interface concavity, which is not favored for crystal quality. On the other hand, the former approach requires a careful control of the local flow. As will be discussed shortly, this will be feasible through ACRT using a novel rotation scheme.

\subsection{Spin-up and spin-down flows and interface morphology}

Computer simulations for the growth using the ACRT schemes in Fig. 2 have been carried out. For the trapezoidal scheme, the flow development in a half-period of a rotation cycle is shown in Fig. 4; the positive stream value indicates a clockwise flow. As compared with the stage indicated in the cycle pattern, it is clearly that $\Psi_{\min }$ is the intensity of the spin-up flow (counterclockwise), while $\Psi_{\max }$ is the intensity of the spin-down flow (clockwise). The directions of spin-up and spin-down flows have been addressed in [11], and the flow structures at stages $A$ and $\mathrm{C}$ in Fig. 4 correspond to the spin-up and spin-down flows, 

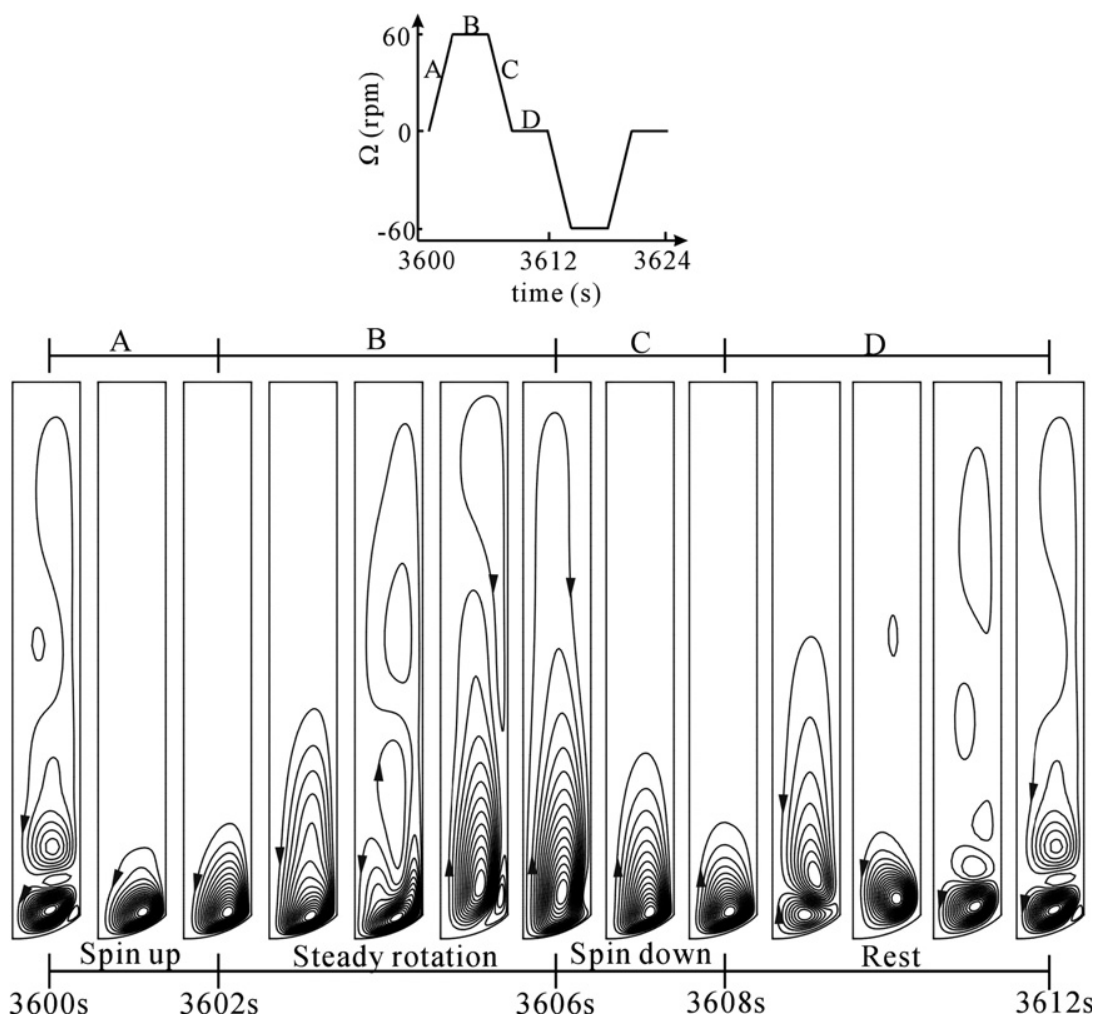

Fig. 4. Flow evolutions at the four stages of the half-period trapezoidal rotation cycle: at $3600 \mathrm{~s}, \Psi_{\min }=-1.964 \times 10^{-3}$ and $\Psi_{\max }=1.678 \times 10^{-4} \mathrm{~g} / \mathrm{s}$; at $3601 \mathrm{~s}, \Psi_{\min }=-9.040 \times 10^{-3}$ and $\Psi_{\max }=1.530 \times 10^{-4} \mathrm{~g} / \mathrm{s} ;$ at $3602 \mathrm{~s}, \Psi_{\min }=-2.478 \times 10^{-2}$ and $\Psi_{\max }=0 \mathrm{~g} / \mathrm{s} ;$ at $3603 \mathrm{~s}, \Psi_{\min }=-9.938 \times 10^{-3}$ and $\Psi_{\max }=0 \mathrm{~g} / \mathrm{s}$; at $3604 \mathrm{~s}, \Psi_{\min }=-2.855 \times 10^{-3}$ and $\Psi_{\max }=3.552 \times 10^{-4} \mathrm{~g} / \mathrm{s}$; at $3605 \mathrm{~s}, \Psi_{\min }=-4.228 \times 10^{-4}$ and $\Psi_{\max }=1.718 \times 10^{-3} \mathrm{~g} / \mathrm{s} ;$ at $3606 \mathrm{~s}$, $\Psi_{\min }=-1.141 \times 10^{-4}$ and $\Psi_{\max }=2.248 \times 10^{-3} \mathrm{~g} / \mathrm{s} ;$ at $3607 \mathrm{~s}, \Psi_{\min }=-7.728 \times 10^{-5}$ and $\Psi_{\max }=2.213 \times 10^{-2} \mathrm{~g} / \mathrm{s} ;$ at $3608 \mathrm{~s}, \Psi_{\min }=-9.195 \times 10^{-5}$ and $\Psi_{\max }=2.751 \times 10^{-2} \mathrm{~g} / \mathrm{s}$; at $3609 \mathrm{~s}, \Psi_{\min }=-7.316 \times 10^{-3}$ and $\Psi_{\max }=6.234 \times 10^{-3} \mathrm{~g} / \mathrm{s}$; at $3610 \mathrm{~s}, \Psi_{\min }=-8.456 \times 10^{-3}$ and $\Psi_{\max }=5.163 \times 10^{-5} \mathrm{~g} / \mathrm{s}$; at $3611 \mathrm{~s}, \Psi_{\min }=-4.241 \times 10^{-3}$ and $\Psi_{\max }=7.798 \times 10^{-4} \mathrm{~g} / \mathrm{s}$; at $3612 \mathrm{~s}, \Psi_{\min }=-1.965 \times 10^{-3}$ and $\Psi_{\max }=1.672 \times 10^{-4} \mathrm{~g} / \mathrm{s}$.

respectively, which are consistent with previous calculations. In stage $\mathrm{B}$, we can see that the flow develops due to the steady rotation, in which a clockwise flow appears, burgeons, and finally dominates the flow direction along the interface. The external spin-up flow is basically caused by the azimuthal acceleration difference of inner and outer radius on the interface. It means, when the interface is rotated under such acceleration, on the interface, liquid near crucible wall is accelerated more than which at the center, this leads an outward flow along the interface to satisfy equation of motion. On the contrary, in the case of spin-down flow, the liquid is decelerated more near crucible wall than at the center, therefore along the interface liquid flows inwardly. The flow during steady rotation is weak and eventually is dominated by the natural convection. Enlightened by the flow reversion in region B, it is believed that the steady rotation increases the developing time of the spin-up flow. Therefore, transposing the steady rotation in the ACRT scheme leads to three types of new schemes shown in Fig. 2c-e.

Fig. 5 shows the averaged flow and acetone fields at $3600 \mathrm{~s}$ under different ACRT schemes. Figs. 5a-d correspond to the scheme shown in Fig. 2b-e, respectively. The averaged flow intensity is calculated by summing stream function value at every time step and divided by the number of time step during one ACRT cycle period. The comparison of the simulated results from Fig. 5 with the observed interface shapes is further shown in Fig. 6. In Fig. 5a, the buoyancy convection cell in the bulk melt is combined with the spin-up flow and this brings acetone into the bulk melt. Due to the better acetone mixing, the acetone accumulation near the interface is less, which can be seen from the smaller $C_{\max } / C_{0}$ (1.993) in the acetone field. No constitutional supercooling is found from the simulation. This is also consistent with the observed interface shape shown in Fig. 6a that no morphological breakdown was observed. On the other hand, the averaged spin-down flow has little effect on the isotherms. The overall isotherms near the interface are distorted inward leading to a more concave interface. This result is similar to the one using a triangular scheme (Fig. 2a), where the interface concavity at the same rotation amplitude is also large. Again the calculated interface concavity $(0.28 \mathrm{~cm})$ is in good agreement with the observed one $(0.24 \mathrm{~cm})$.

With the stair-up ACRT scheme, as shown in Fig. 5b, the interface concavity is much less $(0.21 \mathrm{~cm}$ for the simulation and $0.18 \mathrm{~cm}$ for the observed one) than the previous one $(0.24 \mathrm{~cm})$. However, a small pit appears in the center of the interface. This small pit was also found in the experimental observation shown in Fig. 6b. Apparently, the 

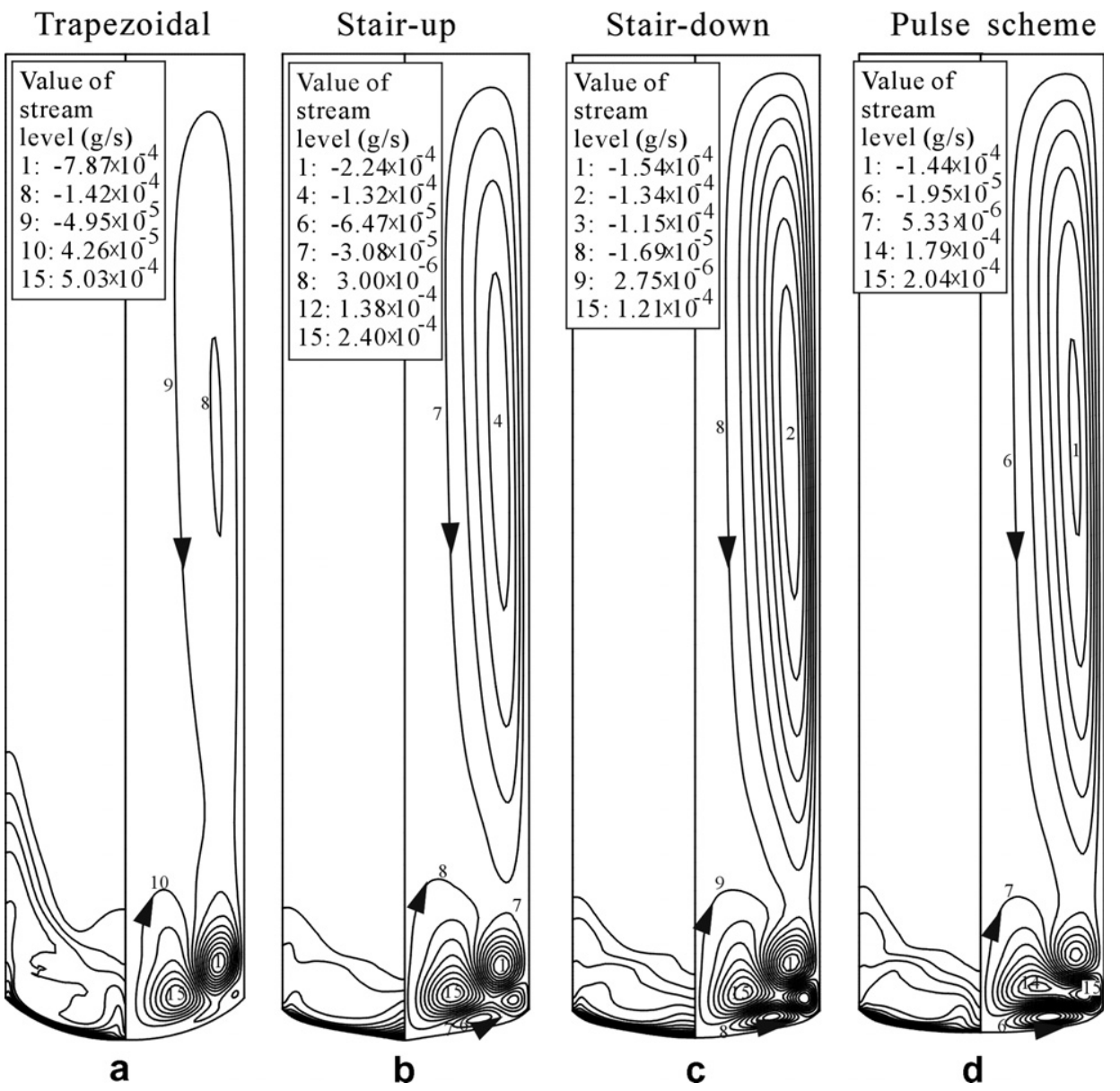

Fig. 5. Averaged solutal (left) and flow (right) fields for different schemes of ACRT at $3600 \mathrm{~s}$. For (a) trapezoidal ACRT, $\Psi_{\min }=-8.789 \times 10^{-4}$, $\Psi_{\max }=5.955 \times 10^{-4} \mathrm{~g} / \mathrm{s}$, and $C / C_{0}=0.996-1.993 ;$ (b) stair-up ACRT, $\Psi_{\min }=-2.677 \times 10^{-4}, \Psi_{\max }=2.737 \times 10^{-4} \mathrm{~g} / \mathrm{s}$, and $C / C_{0}=1.000-3.878,(\mathrm{c})$ stair-down ACRT, $\Psi_{\min }=-1.740 \times 10^{-4}, \Psi_{\max }=1.402 \times 10^{-4} \mathrm{~g} / \mathrm{s}$, and $C / C_{0}=1.000-2.968 ;(\mathrm{d})$ pulse ACRT, $\Psi_{\min }=-1.686 \times 10^{-4}, \Psi_{\max }=2.290 \times$ $10^{-4} \mathrm{~g} / \mathrm{s}$, and $C / C_{0}=0.996-1.993$.

stair-up scheme has one steady rotation period between the spin-up stages, and thus the spin-up flow cannot be fully developed as that in the trapezoidal ACRT scheme. Because the development of the spin-up flow has been retarded, solute accumulates more near the center, which can be seen clearly from the acetone field in Fig. 5b, and this leads to the pit. Interestingly, the flow structure with the stair-down scheme shown in Fig. 5c is similar to that with the stair-up scheme. However, the spin-up flow is slightly enhanced. As a result, the clockwise cell near the interface becomes larger, and this reduces the acetone accumulation at the center of the interface. As a result, no pit is formed there. This is also consistent with the experimental observation shown in Fig. 6c. The interface concavity is further reduced $(0.15 \mathrm{~cm})$; the simulated one is $0.19 \mathrm{~cm}$.

These are evidences that spin-up flow in ACRT can really swipe solute outward from the center and once it has been suppressed, interface morphology will be modified. Nevertheless, the spin-down stage must be in the consequence of spin-up stage, and longer steady rotation after the spin-up flow can induce stronger spin-down one. This is the reason that the stair-up ACRT scheme cannot have a flow strong enough to swipe the solute outward or even to swipe it inward near the interface. If the steady rotation is changed to no rotation, we have the pulse scheme. With this pulse scheme, as shown in Fig. 5d, the spin-up flow is slightly enhanced, so that the interface becomes the flattest among all cases. Nevertheless, because of the longer resting period, the acetone contours can be averaged over the rest stage leading to a more uniform acetone distribution. The simulated interface shape is also in good agreement with the observed one, as shown in Fig. 6d. The calculated interface concavity $(0.16 \mathrm{~cm})$ is also quite close to the observed one $(0.11 \mathrm{~cm})$.

\subsection{Flow intensity integral}

In Fig. 7, an idea called the flow intensity integral (the shaded area) is introduced for better understanding the flow effect on the interface morphology and the solutal redistribution for different ACRT schemes. The four shaded areas for the trapezoidal, stair-up, stair-down, and pulse schemes are shown in Fig. 7a-d, respectively. For the same period, it is clear that the trapezoidal scheme has the largest shaded area, pulse has the smallest, and the other two have the same. During an ACRT cycle, in view 
Experiment

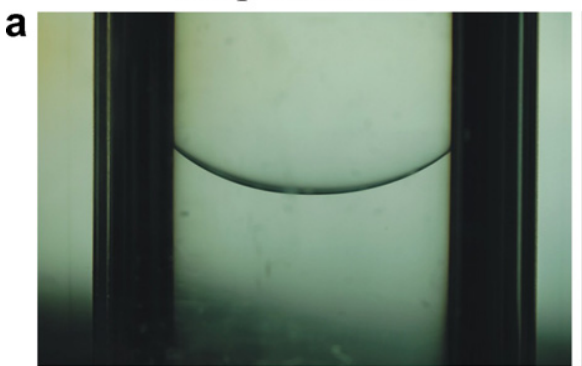

b

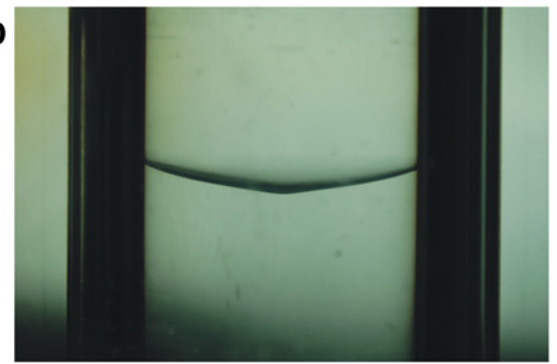

C

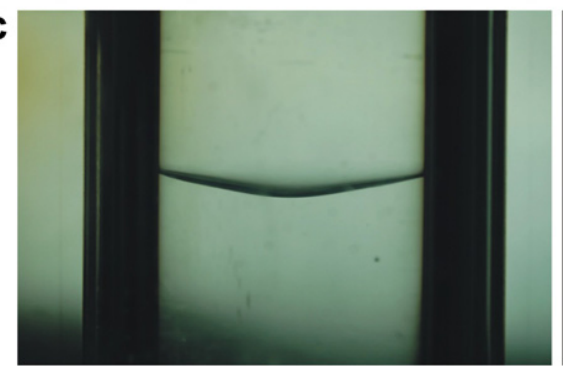

d

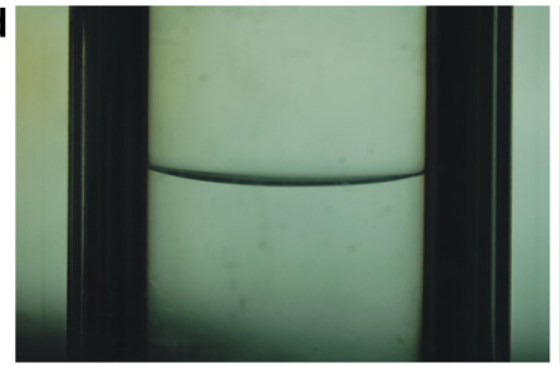

Simulation
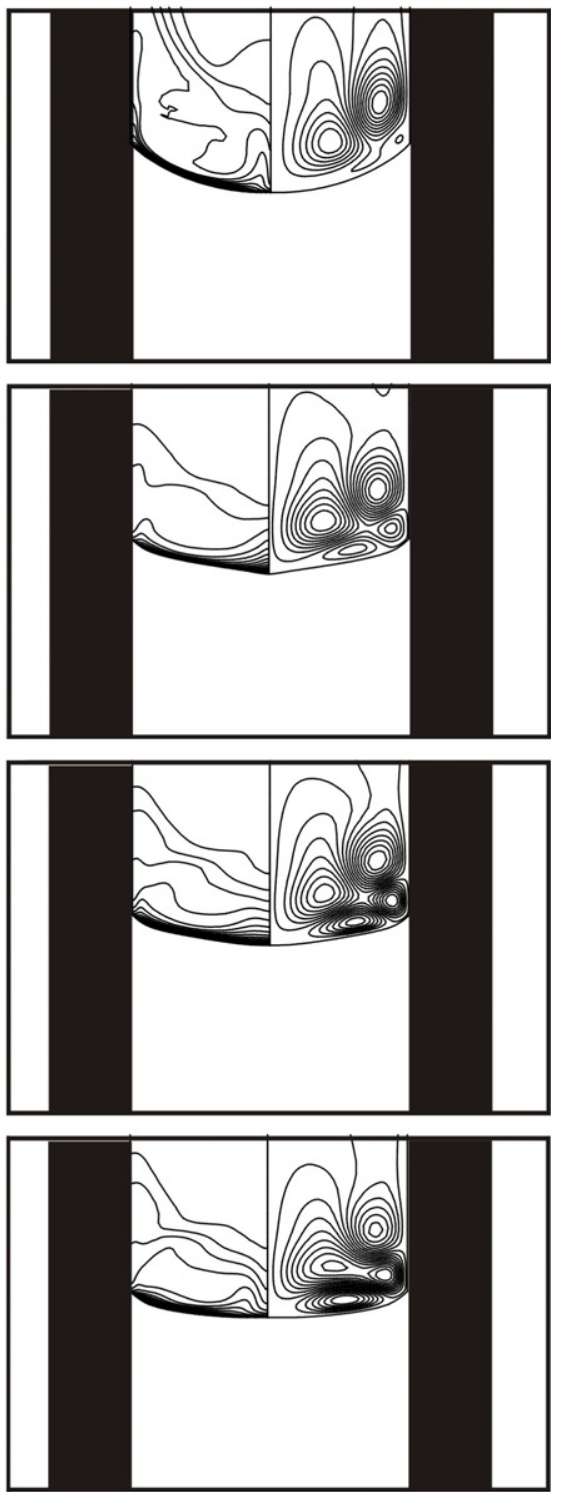

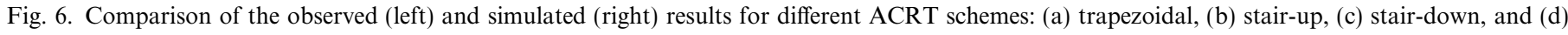
pulse ACRT scheme.

of the flow intensity and the scouring time across the interface, the trapezoidal scheme affects the most on the interface shape. In the cylindrical coordinate, the steady rotation after spin-up allows the Ekman boundary layer to develop. Hence, the thermal and solutal fields, as well as the interface morphology, are better affected. The flow intensity integral means the amount of spin-up and spindown flow and the time for the development of the Ekman boundary layer. Capper et al. [8] and Yeckel and Derby [10] also gave good illustrations of the azimuthal flows in ACRT, and the similar idea is also highlighted here through the flow intensity integral of the azimuthal flow. Because of the difference between tangential velocities on the solid boundary, net streams in the radial direction appear to balance the continuity equation, and thus pump solutes inward and outward during spin-down and spin-up flows, respectively. By comparing the interface concavities for different ACRT schemes in Fig. 5, we can see that larger flow intensity integral leads to a larger interface deformation. And the pulse scheme, having the smallest flow intensity integral, gives the least interface deformation.

\subsection{Radial and axial solute mixing}

Radial concentration profiles along solid-liquid interface for various ACRT schemes are shown in Fig. 8. Without ACRT, as shown, the radial segregation is large, and the acetone accumulates significantly at the center of the interface. With the trapezoidal ACRT scheme, the acetone mixing is significantly enhanced. As a result, the averaged 

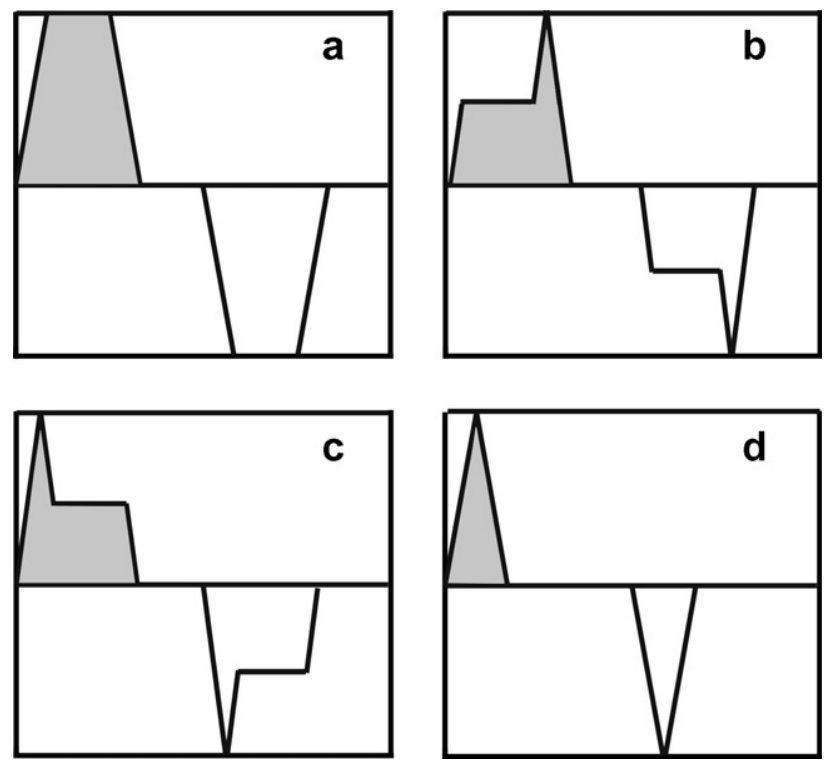

Fig. 7. Illustration of the flow intensity integral (shaded area) for (a) trapezoidal, (b) stair-up, (c) stair-down, and (d) pulse ACRT schemes.

concentration near the interface is significantly lower than others. On the other hand, for the stair-up and stair-down schemes, as mentioned previously, the development of the spin-up flow is retarded, so that the spin-down and thermal convection are still dominant near the center of the interface. As a result, the solute still accumulates slightly more near the center of the interface; nevertheless, they are still significantly better than that of no ACRT. As compared

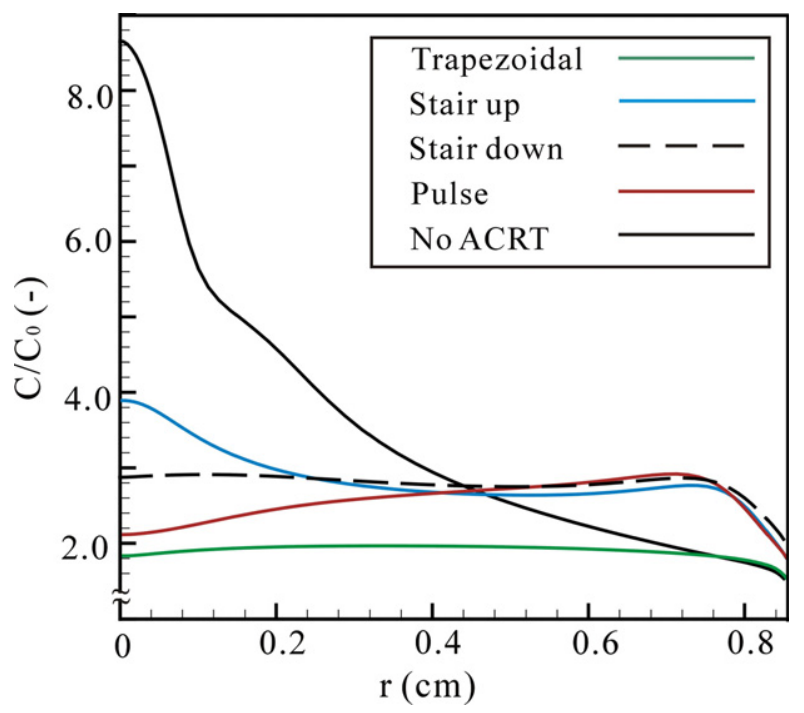

Fig. 8. Radial acetone profiles for four different ACRT schemes at $3600 \mathrm{~s}$.

with the radial concentration of the stair-up and stair-down schemes, the pulse scheme results in a much lower concentration near center. This is simply due to the development of the spin-up flow is avoided, while the spin-down flow is allowed to developed due to the longer rest time. On the other hand, because the flow intensity integral is smaller, the global mixing is not enhanced much; the interface morphology is not affected much as well. Therefore, the average concentration is still much higher than that of the trapezoidal scheme.
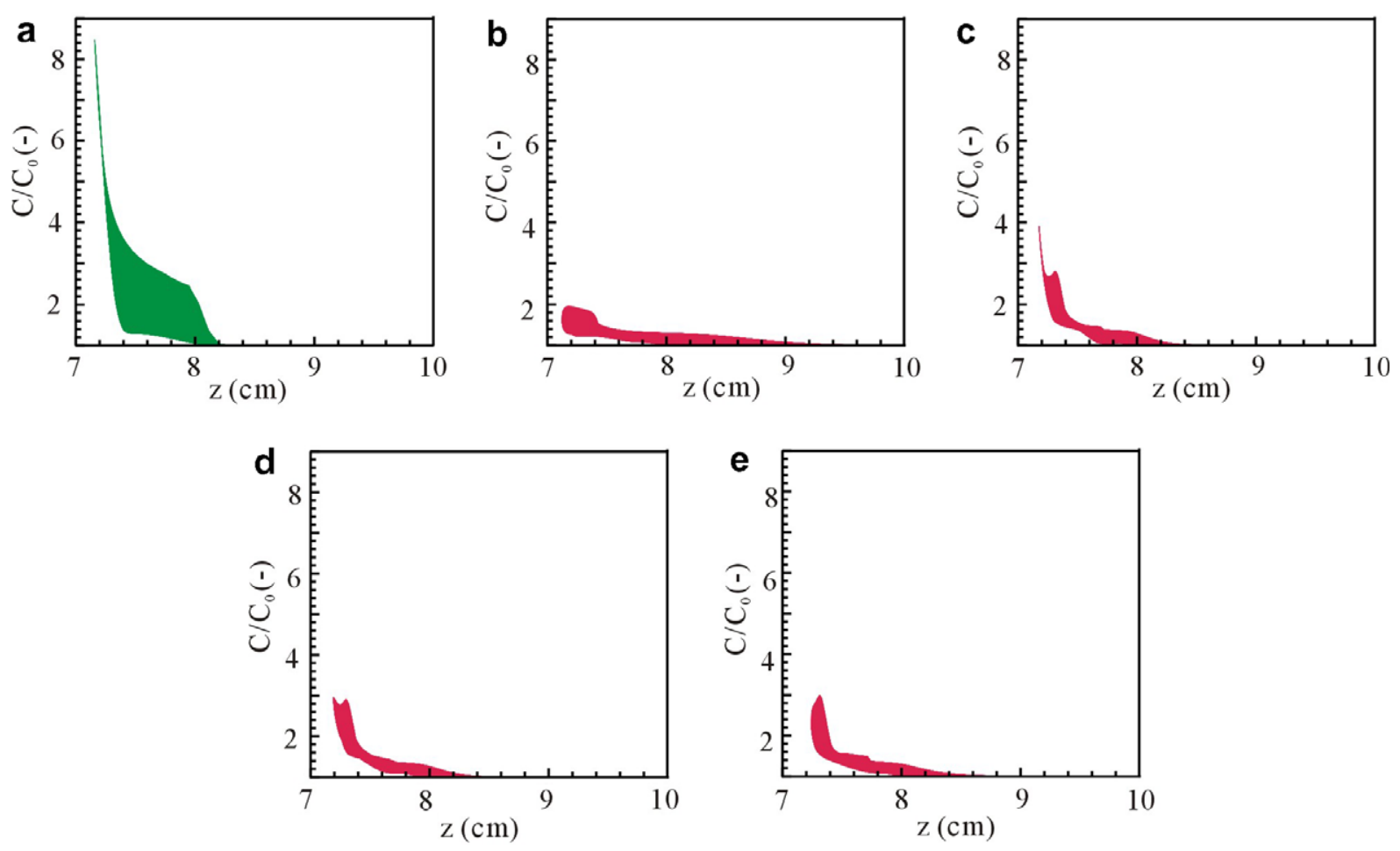

Fig. 9. Axial acetone distributions in the melt for four different ACRT schemes at $3600 \mathrm{~s}$; for (a) without ACRT, (b) trapezoidal, (c) stair-up, (d) stairdown, and (e) pulse ACRT scheme. 
To further illustrate the effect of the ACRT schemes on the global mixing. The calculated axial acetone concentration variations in the melt at $3600 \mathrm{~s}$ are plotted in Fig. 9. As shown, the trapezoidal scheme gives a much wider distribution in the axial direction. For the stair-up, stair-down, and pulse schemes, the axial solute distributions are about the same, except a much higher concentration near the interface for the stair-up scheme, which is caused by the larger radial segregation. From the axial segregation point of view, the less global segregation is favorable for the reduction of the axial segregation if the growth length is significantly longer than the diffusion boundary layer.

\section{Conclusion}

We have demonstrated that the ACRT schemes can significantly affects melt flow, interface morphology, and axial and radial solute segregations during vertical Bridgman crystal growth. The traditional triangular and trapezoidal schemes are effective in controlling the radial segregation if the rotation amplitude is high enough. However, this often results in a severe interface deformation and a larger axial segregation, as a result of the global solute mixing. Three new schemes are proposed in this study. It is found that the pulse scheme, which enhances the spin-down flow, seems to be an effective way in reducing radial solute segregation, while the interface deformation can be minimized. These simulated results are further confirmed by the experimental observations.

\section{Acknowledgement}

This work was sponsored by the National Science Council (NSC) of Taiwan and the Orchid program between NSC and France Science Foundation.

\section{References}

[1] H.J. Scheel, E.O. Schulz-Dubois, J. Crystal Growth 8 (1971) 304.

[2] P. Capper, J.J.G. Gosney, C.L. Jones, J. Crystal Growth 70 (1984) 356.

[3] W.G. Coates, P. Capper, C.L. Jones, J.J.G. Gosney, C.K. Ard, I. Kenworthy, A. Clark, J. Crystal Growth 94 (1989) 959.

[4] P. Capper, J.J.G. Gosney, C.L. Jones, I. Kenworthy, J. Electron. Mater. 15 (1986) 371.

[5] P. Capper, W.G. Coates, C.L. Jones, J.J.G. Gosney, C.K. Ard, I. Kenworthy, J. Crystal Growth 83 (1987) 69.

[6] P. Capper, J.C. Brice, C.L. Jones, W.G. Coates, J.J.G. Gosney, C.K. Ard, I. Kenworthy, J. Crystal Growth 89 (1988) 171.

[7] R.U. Bloedner, P. Gille, J. Crystal Growth 130 (1993) 181.

[8] P. Capper, C. Maxey, C. Butler, M. Grist, J. Price, J. Crystal Growth 275 (2005) 259.

[9] J.C. Brice, Crystal Growth Processes, Wiley, New York, 1986.

[10] A. Yeckel, J.J. Derby, J. Crystal Growth 209 (2000) 734.

[11] A. Yeckel, J.J. Derby, J. Crystal Growth 233 (2001) 599.

[12] X. Liu, W. Jie, Y. Zhou, J. Crystal Growth 219 (2000) 22.

[13] Y.C. Liu, B. Roux, C.W. Lan, J. Crystal Growth, under revision.

[14] C.W. Lan, M.C. Liang, J. Crystal Growth 186 (1998) 203.

[15] Y.C. Liu, W.C. Yu, B. Roux, T.P. Lyubimova, C.W. Lan, Chem. Eng. Sci. 61 (2006) 7766.

[16] C.W. Lan, Y.W. Yang, C.Y. Tu, J. Crystal Growth 235 (2002) 619.

[17] C.W. Lan, J. Crystal Growth 274 (2005) 379. 